\title{
Image-charge effects on the envelope dynamics of an unbunched intense charged-particle beam
}

\author{
B. L. Qian, ${ }^{*}$ J. Zhou, and C. Chen \\ Plasma Science and Fusion Center, Massachusetts Institute of Technology, Cambridge, Massachusetts 02139
}

(Received 5 August 2002; published 6 January 2003)

\begin{abstract}
The root-mean-squared (rms) envelope equations are derived and analyzed for an unbunched intense charged-particle beam in an alternating-gradient focusing field and a cylindrical conducting pipe. All higher-order image-charge effects from the cylindrical pipe are expressed in terms of so-called multiple moment factors in the rms beam envelope equations, and the multiple moment factors are evaluated. Numerical results show that for vacuum phase advance $\sigma_{v}<90^{\circ}$, the image-charge effects on the matched and slightly mismatched beam envelopes are negligibly small, at all orders, for all beams with arbitrary beam density profiles (including hollow density profiles) as well as for arbitrary small apertures (including beams with large aspect ratios). However, the main unstable region for the envelope evolution with image-charge effects, which occurs for $90^{\circ}<\sigma_{v}<270^{\circ}$, depending on the value of the normalized beam intensity $S K / \varepsilon$, is found to be narrower than its counterpart without image-charge effects.
\end{abstract}

DOI: $10.1103 /$ PhysRevSTAB.6.014201

PACS numbers: 41.85.Ja, 41.75.-i

\section{INTRODUCTION}

High-intensity accelerators with alternating-gradient focusing systems have many applications in basic scientific research and nuclear physics. These applications include heavy ion fusion [1,2], nuclear waste treatment [3], and spallation neutron source [4]. In the research and development of high-intensity accelerators, a key issue is to minimize the aperture of the transport system for intense charged-particle beams, while preventing the beams from developing large-amplitude charge density and velocity fluctuations as well as subsequent emittance growth and halo formation [4-15]. In order to understand the collective behavior of charged-particle beams, it is important to examine the beam envelope evolution under the influence of both the beam space charge and the image charges induced on the conducting walls of accelerator structures.

Intense charged-particle beams in alternating-gradient focusing systems have been investigated since the late 1950s. One of the earliest works on the beam envelope equations can be found in Ref. [16], in which the wellknown two-dimensional (2D) Kapchinskij-Vladimirskij $(\mathrm{KV})$ equations were derived for a uniform beam density in free space. In 1965, Lapostolle [17] gave the threedimensional (3D) envelope equations for a uniform beam density in free space, and his results could be applicable for bunched beams. In 1971, Sacherer [18] and Lapostolle [19] extended the previous $2 \mathrm{D}$ and $3 \mathrm{D}$ results to the arbitrary beam density cases in free space. Sacherer's 2D results, which describe the root-mean-squared (rms) beam envelopes for all elliptical beams with arbitrary beam densities, assume the same form of the KV equations.

Recently, Allen and Reiser [20,21] extended Sacherer's $2 \mathrm{D}$ results to include the image-charge effects due to the cylindrical conducting pipe. They analyzed the first-order image-charge effects. Their results showed that the firstorder image-charge effects do not affect the beam envelope evolution until the beam is very close to the wall of the cylindrical conducting pipe. The expressions for the first-order image-charge contributions in the rms beam envelope equations in $[20,21]$ were identical to those obtained in an earlier paper by Lee, Close, and Smith [22].

In general, there are two approaches in treating the density distribution of a nonequilibrium beam. One approach uses a rigorous normal-mode analysis (i.e., smallsignal theory) $[23,24]$ to describe the density evolution in a charged-particle beam. The normal-mode analysis is useful for understanding the collective oscillations and instabilities in the beam. The other approach makes use of the assumption of a self-similar beam density distribution [7-9,11,17-21]. While the self-similar beam density distribution is not rigorous and, in general, not selfconsistent, it has proven to be a useful model in the many previous derivations of rms beam envelope equations [7-9,11,17-21]. The rms beam envelope equations based on the self-similar model are very useful in the design of beam optics. Although the beam density usually does not remain in the prescribed self-similar form, the rms envelope equations still give a quite accurate description of an intense beam, provided the actual beam emittances, which can be either measured in the beam experiment or computed in the self-consistent simulation, are used (see, for example, Ref. [25]).

In this paper, we extend Allen and Reiser's 2D envelope equations to include all higher-order image-charge effects from the cylindrical conducting pipe. In particular, the self-electric and self-magnetic fields are calculated for an unbunched beam with elliptic symmetry and an 
arbitrary transverse dependence in the self-similar beam density model [7-9,11,17-21]. The rms envelope equations are derived, including all higher-order imagecharge effects, from the cylindrical conducting pipe. Numerical results show that for vacuum phase advance $\sigma_{v}<90^{\circ}$, the image-charge effects on the matched and slightly mismatched beam envelopes are negligibly small, at all orders, for all beams with arbitrary beam density profiles (including hollow density profiles measured recently in the heavy ion injector experiment at Lawrence Berkeley National Laboratory [26]) as well as for arbitrary small apertures (including beams with large aspect ratios). However, the main unstable region for the envelope with image-charge effects, which occurs for $90^{\circ}<\sigma_{v}<270^{\circ}$, depending on the value of the normalized beam intensity $S K / \varepsilon$, is found to be narrower than its counterpart without image-charge effects [11].

The organization of the present paper is as follows. In Sec. II, 2D envelope equations are derived, including all higher-order image-charge effects. In Sec. III, the multiple moment factors in the envelope equations are evaluated. In Sec. IV, the higher-order image-charge effects on the matched beam envelopes are investigated by numerically solving the 2D envelope equations for arbitrary beam densities, including a hollow beam density profile, in a cylindrical conducting pipe. In Sec. V, the image-charge effects on the slightly mismatched beams and beam envelope instability are numerically analyzed. Conclusions are presented in Sec. VI.

\section{BEAM ENVELOPE EQUATIONS WITH IMAGE-CHARGE EFFECTS}

We consider an unbunched elliptical beam propagating in an alternating-gradient focusing field and a cylindrical metal pipe with radius $R$, as shown in Fig. 1. The beam has an envelope $a(s)$ in the $x$ direction and an envelope

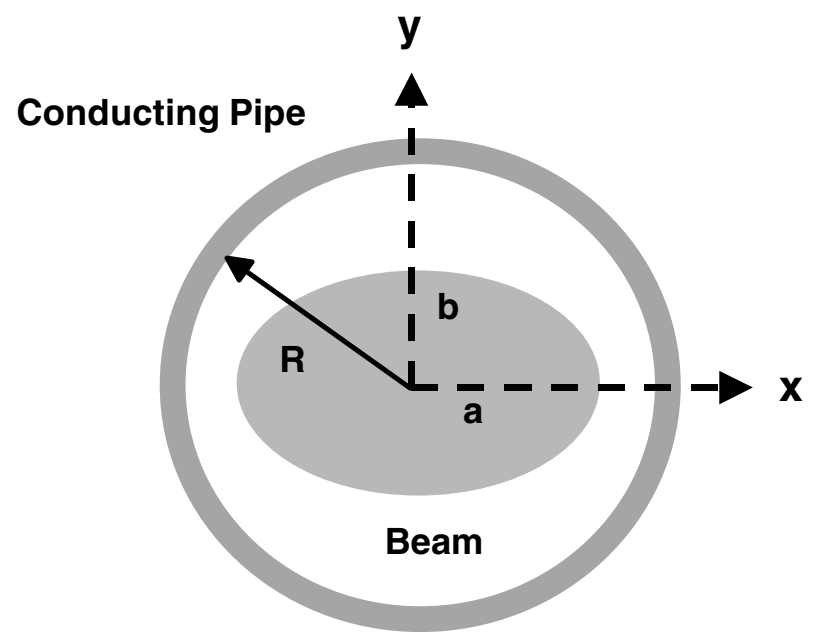

FIG. 1. Elliptical unbunched charged-particle beam in a cylindrical conducting pipe. $b(s)$ in the $y$ direction, where $s=z$. The beam drift velocity in the $z$ direction is $v_{z} \approx \beta_{b} c$, where $c$ is the speed of light in vacuum. In the present analysis, we assume that the beam centroid motion is stable and on the $z$ axis, and ignore the beam centroid motion. Following the analysis of Sacherer [18], we express the beam density in a self-similar beam model as

$$
n(x, y, s)=n\left(\frac{x^{2}}{a^{2}}+\frac{y^{2}}{b^{2}}\right)
$$

The general rms envelope equations can be expressed as [18]

$$
\frac{d^{2} \tilde{x}}{d s^{2}}+\kappa_{q}(s) \tilde{x}+\frac{K}{2 q N_{b} \tilde{x}}\left\langle x \frac{\partial \phi}{\partial x}\right\rangle=\frac{1}{\tilde{x}^{3}}\left(\frac{\varepsilon_{x}}{4}\right)^{2}
$$

and

$$
\frac{d^{2} \tilde{y}}{d s^{2}}-\kappa_{q}(s) \tilde{y}+\frac{K}{2 q N_{b} \tilde{y}}\left\langle y \frac{\partial \phi}{\partial y}\right\rangle=\frac{1}{\tilde{y}^{3}}\left(\frac{\varepsilon_{y}}{4}\right)^{2} .
$$

In Eqs. (2) and (3), \langle\rangle denotes the moment operator with respect to the particle beam distribution; $\tilde{x}=\sqrt{\left\langle x^{2}\right\rangle}$ and $\tilde{y}=\sqrt{\left\langle y^{2}\right\rangle}$ are the rms envelopes in the $x$ and $y$ directions, respectively;

$$
\varepsilon_{x}=4\left[\left\langle x^{2}\right\rangle\left\langle(d x / d s)^{2}\right\rangle-\langle x(d x / d s)\rangle^{2}\right]^{1 / 2},
$$

and

$$
\varepsilon_{y}=4\left[\left\langle y^{2}\right\rangle\left\langle(d y / d s)^{2}\right\rangle-\langle y(d y / d s)\rangle^{2}\right]^{1 / 2}
$$

are 4 times the beam rms emittances in the $x$ and $y$ directions, respectively; $s=c \beta_{b} t$ is the axial distance; $\phi$ is the potential distribution generated by the beam space charge including image-charge effects of the cylindrical conducting pipe; $\kappa_{q}(s)=q B_{q}^{\prime}(s) /\left(\gamma_{b} m \beta_{b} c\right)$ is the focusing parameter of the alternating-gradient focusing system; $\vec{B}^{q}=B_{q}^{\prime}(s)(y \hat{x}+x \hat{y})$ is the alternatinggradient quadrupole magnetic field with $B_{q}^{\prime}(s)=B_{q}^{\prime}(s+$ $S$ ), where $S$ is the period of the field, $m$ and $q$ are the rest mass and charge of the particle, respectively; $K=$ $2 q^{2} N_{b} / \gamma^{3} m \beta_{b}^{2} c^{2}$ is the generalized beam perveance; $N_{b}=\int_{-\infty}^{\infty} \int_{-\infty}^{\infty} n(x, y, s) d x d y$ is the number of charged particle per unit length along the $z$ direction; $\gamma=(1-$ $\left.\beta_{b}^{2}\right)^{-1 / 2}$ is the relativistic factor of the beam.

In order to derive an explicit expression for the potential $\phi$, we solve Poisson's equation

$$
\frac{1}{r} \frac{\partial}{\partial r}\left(r \frac{\partial \phi}{\partial r}\right)+\frac{1}{r^{2}} \frac{\partial^{2} \phi}{\partial \theta^{2}}=-4 \pi q n\left(\frac{x^{2}}{a^{2}}+\frac{y^{2}}{b^{2}}\right)
$$

under the paraxial approximation with the boundary condition $\left.\phi\right|_{r=R}=0$. Here $(r, \theta, z)$ is the corresponding cylindrical coordinate system. Using Green's function technique, it is readily shown that the solution to Eq. (6) can be expressed as

$$
\phi=\phi^{\text {free }}+\phi^{\text {image }},
$$


where

$$
\phi^{\text {free }}=-\pi a b q \int_{0}^{\infty} \frac{d u}{\sqrt{\left(a^{2}+u\right)\left(b^{2}+u\right)}} \int_{0}^{T} n\left(T^{\prime}\right) d T^{\prime}
$$

is the free-space contribution of the beam,

$$
\begin{aligned}
\phi^{\text {image }}= & 2 q \int_{0}^{\infty} \int_{0}^{2 \pi} \ln \left(\frac{R}{r^{\prime}}\right) n\left(\frac{r^{\prime 2} \cos ^{2} \theta^{\prime}}{a^{2}}+\frac{r^{\prime 2} \sin ^{2} \theta^{\prime}}{b^{2}}\right) r^{\prime} d r^{\prime} d \theta^{\prime} \\
& -2 q \sum_{m=1}^{\infty} \int_{0}^{\infty} \int_{0}^{2 \pi} \frac{1}{m}\left(\frac{r^{\prime} r}{R^{2}}\right)^{m} \cos \left[m\left(\theta-\theta^{\prime}\right)\right] n\left(\frac{r^{\prime 2} \cos ^{2} \theta^{\prime}}{a^{2}}+\frac{r^{\prime 2} \sin ^{2} \theta^{\prime}}{b^{2}}\right) r^{\prime} d r^{\prime} d \theta^{\prime}
\end{aligned}
$$

is the image-charge contribution from the cylindrical metal pipe, and the function $T$ is defined by

$$
T=\frac{x^{2}}{a^{2}+u}+\frac{y^{2}}{b^{2}+u} .
$$

The space-charge terms in the envelope equations can be simplified by using Eqs. (7)-(10). The results are

$$
\left\langle x \frac{\partial \phi}{\partial x}\right\rangle=N_{b} q\left(I_{x}^{\mathrm{free}}+I_{x}^{\mathrm{image}}\right),
$$

and

$$
\left\langle y \frac{\partial \phi}{\partial y}\right\rangle=N_{b} q\left(I_{y}^{\mathrm{free}}+I_{y}^{\mathrm{image}}\right)
$$

where

$$
I_{x}^{\mathrm{free}}=-\frac{\tilde{x}}{\tilde{x}+\tilde{y}}
$$

and

$$
I_{y}^{\mathrm{free}}=-\frac{\tilde{y}}{\tilde{x}+\tilde{y}}
$$

are the space-charge contributions in free space [18], and

$$
I_{x}^{\text {image }}=-\sum_{l=1}^{\infty} \frac{\tilde{x}^{2}\left(\tilde{x}^{2}-\tilde{y}^{2}\right)^{2 l-1}}{R^{4 l}} 4^{2 l} N_{l}
$$

and

$$
I_{y}^{\text {image }}=-\sum_{l=1}^{\infty} \frac{\tilde{y}^{2}\left(\tilde{y}^{2}-\tilde{x}^{2}\right)^{2 l-1}}{R^{4 l}} 4^{2 l} N_{l}
$$

are the image-charge contributions from the cylindrical conducting pipe. Here,

$$
N_{l}=2\left(\frac{(2 l) !}{4^{l}(l !)^{2}}\right)^{2} \frac{\left[\int_{0}^{\infty} 2 \pi a b n\left(\hat{r}^{2}\right) \hat{r}^{2 l+1} d \hat{r}\right]^{2}\left[\int_{0}^{\infty} 2 n\left(\hat{r}^{2}\right) \hat{r} d \hat{r}\right]^{2 l}}{N_{b}^{2}\left[4 \int_{0}^{\infty} n\left(\hat{r}^{2}\right) \hat{r}^{3} d \hat{r}\right]^{2 l}}
$$

is a multiple moment factor related to the beam density profile.

Substituting Eqs. (11)-(17) into Eqs. (2) and (3), we obtain the envelope equations of the following form:

$$
\frac{d^{2} X}{d s^{2}}+\kappa_{q}(s) X-2 K\left[\frac{1}{X+Y}+\sum_{l=1}^{\infty} \frac{X\left(X^{2}-Y^{2}\right)^{2 l-1}}{R^{4 l}} N_{l}\right]=\frac{\varepsilon_{x}^{2}}{X^{3}}
$$

and

$$
\frac{d^{2} Y}{d s^{2}}-\kappa_{q}(s) Y-2 K\left[\frac{1}{X+Y}+\sum_{l=1}^{\infty} \frac{Y\left(Y^{2}-X^{2}\right)^{2 l-1}}{R^{4 l}} N_{l}\right]=\frac{\varepsilon_{y}^{2}}{Y^{3}},
$$

where $X=2 \tilde{x}$ and $Y=2 \tilde{y}$.

Unlike the previous results obtained by Allen and Reiser [20,21], which include only the $l=1$ contribution, the present envelope Eqs. (18) and (19) are complete, including both the $l=1$ contribution and all of the higher-order image-charge effects with $l \geq 2$.

\section{EVALUATION OF MULTIPLE MOMENT FACTORS}

The multiple moment factor $N_{l}$ contains the information about the higher-order image-charge effects in the envelope equations (18) and (19). We can assess these effects by evaluating $N_{l}$ as a function of $l$. In particular, we consider the following parabolic density profile [11]:

$$
n= \begin{cases}n_{0}+\delta n_{0}\left[1-3\left(\frac{x^{2}}{a^{2}}+\frac{y^{2}}{b^{2}}\right)^{2}\right], & \frac{x^{2}}{a^{2}}+\frac{y^{2}}{b^{2}} \leq 1, \\ 0, & \frac{x^{2}}{a^{2}}+\frac{y^{2}}{b^{2}}>1\end{cases}
$$

where

$$
N_{b}=\int_{-\infty}^{\infty} \int_{-\infty}^{\infty} n d x d y=\pi a b n_{0}=\mathrm{const},
$$


and $\delta n_{0}$ is independent of $x$ and $y$ and satisfies $-n_{0} \leq$ $\delta n_{0} \leq n_{0} / 2$.

In Eq. (20), $\delta n_{0}=0$ means that the beam density is a constant across the beam profile, $\delta n_{0}>0$ indicates that the beam density decreases monotonically from the center of profile to its edge and represents a Gaussian-like beam profile, and $\delta n_{0}<0$ implies that the beam density increases monotonically from the center of beam profile to its edge and represents a hollow beam profile such as that observed in the heavy ion injector experiment at LBNL [26].

Using Eq. (20) and the moment definition, we can obtain the simplified expressions of envelopes $X$ and $Y$. They are written in the form of

$$
X^{2}=a^{2}\left(1-\frac{1}{2} g\right)
$$

and

$$
Y^{2}=b^{2}\left(1-\frac{1}{2} g\right)
$$

In addition, substituting Eqs. (20) and (21) into Eq. (17), we obtain

$$
N_{l}=2\left(\frac{(2 l) !}{4^{l}(l !)^{2}}\right)^{2}\left(\frac{1}{l-0.5 g}\right)^{2 l}\left(\frac{1-2 g l /(l+3)}{l+1}\right)^{2}
$$

where

$$
g=\frac{\delta n_{0}}{n_{0}}
$$

Note in Eq. (24) that $N_{1}=1 / 8=0.125$ is independent of the factor $g$.

Figure 2 shows a plot of $N_{l}$ as a function of $l$ for three cases corresponding to $g=0,0.5$, and -0.5 . In Fig. 2, $N_{1}=0.125$ for all three cases, as expected. For $l \geq 2$,

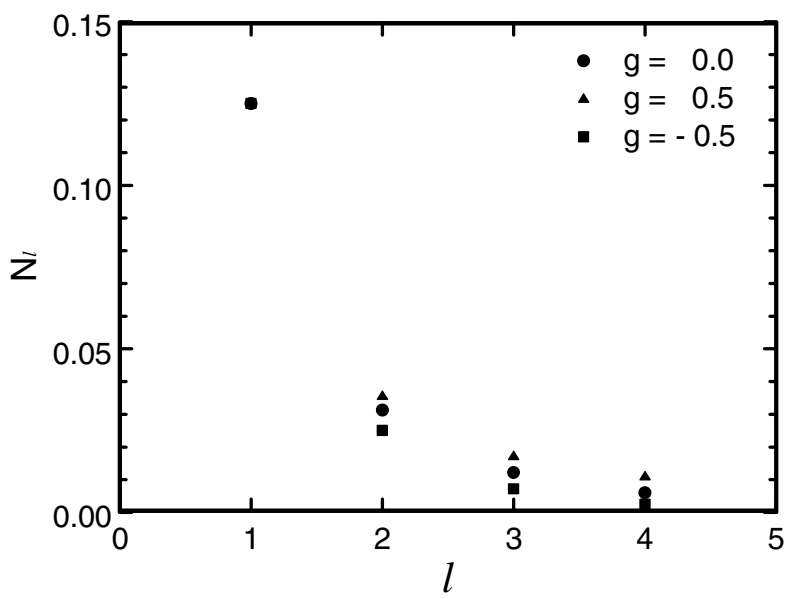

FIG. 2. The dimensionless multiple moment factor $N_{l}$ versus $l$ for several densities with $g=0,0.5$, and -0.5 . however, the value of $N_{l}$ decreases with increasing $l$ and depends strongly on the factor $g$ (i.e., on the choice of the density profile). In addition, for a given value of $l>1$, $N_{l}$ increases with $g$; that is, the value of $N_{l}$ is lower at $g=-0.5$ than at $g=0.5$. However, this does not necessarily mean that the image-charge effects of a hollow beam are weaker than those of a solid beam because the beam envelopes $X$ and $Y$ given by Eqs. (22) and (23) increase considerably with decreasing $g$. The physics of image-charge effects is contained in $X$ and $Y$. Obviously, the values of $X$ and $Y$ are higher at $g=-0.5$ than at $g=0.5$, which implies that the image-charge effects of a hollow beam are stronger than those of a solid beam.

\section{IMAGE-CHARGE EFFECTS ON RMS MATCHED BEAMS}

In this section, we investigate numerically the imagecharge effects on a matched beam using the analytical results obtained in Sec. II, and show that for vacuum phase advance $\sigma_{v}<90^{\circ}$, the higher-order image-charge effects on the matched beam envelopes are negligibly small for all beams with arbitrary beam density profiles (including hollow density profiles) as well as for arbitrary small apertures (including beams with large aspect ratios). We pay special attention to a hollow beam observed in a recent heavy ion beam experiment [26].

For present purposes, we assume that the rms emittances $\varepsilon_{x}$ and $\varepsilon_{y}$ are constant, i.e., $\varepsilon_{x}=\varepsilon_{y}=\varepsilon$, and that the beam density profile is given in Eq. (20). We also assume that the alternating-gradient transport system is presented a step-function lattice (in Fig. 3) defined by [11]

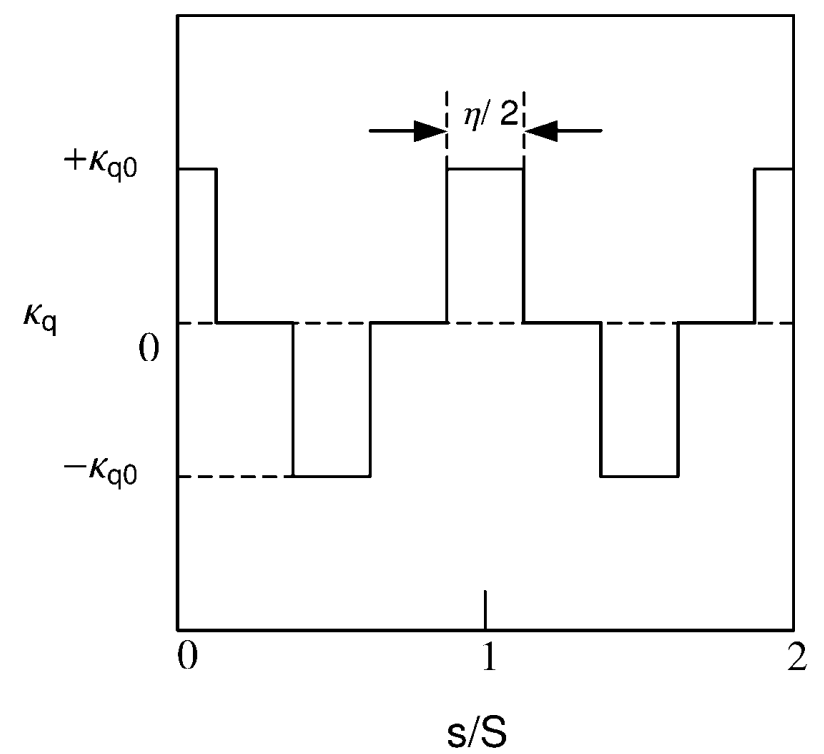

FIG. 3. Plot of the normalized step-function lattice $\kappa_{q}(s)$ versus normalized propagating distance $s / S$ for $\eta=0.5$. 


$$
\kappa_{q}(s)= \begin{cases}+\kappa_{q 0}, & 0 \leq s / S<0.25 \eta \\ 0, & 0.25 \eta \leq s / S<0.5(1-0.5 \eta) \\ -\kappa_{q 0}, & 0.5(1-0.5 \eta) \leq s / S<0.5(1+0.5 \eta), \\ 0, & 0.5(1+0.5 \eta) \leq s / S<0.5(2-0.5 \eta) \\ +\kappa_{q 0}, & 0.5(2-0.5 \eta) \leq s / S<1\end{cases}
$$

where $\kappa_{q 0}$ is a constant and $\eta(0<\eta<1)$ is the filling factor. The strength of the alternating-gradient focusing field can be measured in terms of the vacuum phase advance $\sigma_{v}$. For step-function lattice defined in Eq. (26), the vacuum phase advance $\sigma_{v}$ satisfies [11]

$$
\begin{aligned}
\cos \sigma_{v}= & \cosh \varphi_{1}\left(\cos \varphi_{1}-\varphi_{2} \sin \varphi_{1}\right) \\
& +\varphi_{2} \sinh \varphi_{1}\left(\cos \varphi_{1}-0.5 \varphi_{2} \sin \varphi_{1}\right),
\end{aligned}
$$

where

$$
\varphi_{1}=0.5 S \eta \sqrt{\kappa_{q 0}}
$$

and

$$
\varphi_{2}=\frac{1-\eta}{\eta} \varphi_{1}
$$

In the numerical analysis of the beam envelope equations (18) and (19), it is convenient to use the dimensionless parameters and normalized variables defined by $\hat{s}=s / S, \quad \tilde{a}=X / \sqrt{S \varepsilon}, \quad \tilde{b}=Y / \sqrt{S \varepsilon}, \quad \hat{K}=K S / \varepsilon, \quad \hat{R}=$ $R / \sqrt{S \varepsilon}$, and $\tilde{\kappa}_{q}(s)=S^{2} \kappa_{q}(s)$. For example, Fig. 4 shows the matched beam envelope functions $\tilde{a}(s)=\tilde{a}(s+S)$ and $\tilde{b}(s)=\tilde{b}(s+S)$ for $\sigma_{v}=80^{\circ}, \hat{K}=10, \eta=0.5, g=$ -0.5 for beam propagation in free space as well as in a cylindrical conducting pipe with $\hat{R}=4$.0. It is evident in Fig. 4 that the image-charge effects, including the contributions from all orders, are negligibly small for a hollow beam whose maximum envelopes are very close

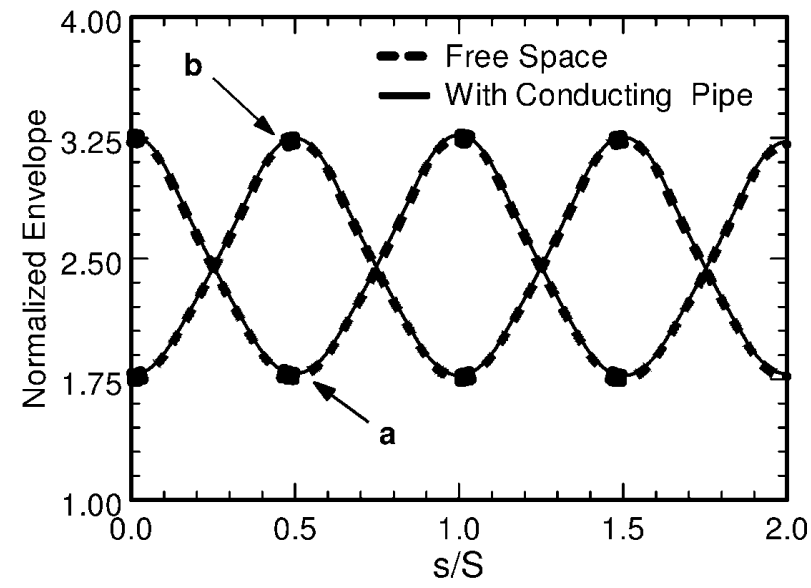

FIG. 4. Plot of the normalized envelope functions $\tilde{a}$ and $\tilde{b}$ versus normalized propagating distance $s / S$ for $\sigma_{v}=80^{\circ}, \hat{K}=$ $10, g=-0.5, \eta=0.5$, and $\hat{R}=4$. to the wall of the cylindrical pipe ( $\tilde{a}_{\max }=\tilde{b}_{\max } \approx 3.25$ and $\hat{R}=4.0$ ).

We have carried out comprehensive numerical studies of the image-charge effects on the matched envelope functions for different beam density profiles, including beams with large aspect ratios. The numerical results show that for vacuum phase advance $\sigma_{v}<90^{\circ}$, the total image-charge effects, including higher-order ones, do not affect significantly the matched beam envelopes for arbitrary beam density profiles and arbitrarily small apertures (conducting pipes).

\section{IMAGE-CHARGE EFFECTS ON SLIGHTLY MISMATCHED BEAMS AND ENVELOPE INSTABILITIES}

In a real device, it is almost impossible to obtain a precisely matched beam because there exist some perturbations on the beam propagation. These perturbations may cause beam envelope instabilities, and the unstable beam envelopes may result in particle beam losses. A perturbation on the matched beam results in a mismatched beam in the alternating-gradient focusing system. The beam envelope instability has already been investigated in free space [11-15]. However, the imagecharge effects of the cylindrical conducting pipe on the mismatched beams and the beam envelope instability have not been studied until the present paper. In this section, the envelope equations (18) and (19) are solved, assuming $\varepsilon_{x}=\varepsilon_{y}=\varepsilon$, for slightly mismatched beams to find the unstable regions in the parameter space.

In order to obtain slightly mismatched beam envelopes, following the method employed in Ref. [11], the initial conditions for $\tilde{a}(s)$ and $\tilde{b}(s)$ at $s=0$ are chosen to be

$$
\tilde{a}(0)=\tilde{a}_{0}(1+\delta)
$$

and

$$
\tilde{b}(0)=\tilde{b}_{0}(1-\delta),
$$

where $\tilde{a}_{0}$ and $\tilde{b}_{0}$ are the matched beam envelopes at $s=$ 0 , and $\delta$ is chosen to be in the region of $0 \leq|\delta| \leq 0.01$. Here, $\delta$ represents the perturbations caused by the noise in the beam. Therefore, the initial perturbation to the matched beam envelope is assumed to be as small as $1 \%$. In the numerical calculations, the particle beams are allowed to propagate over 40 periods. Further increasing the number of the propagation periods does not affect the determination of the unstable regions. In order to determine the unstable regions for beam envelopes, we run the envelope code and observe if the beam envelopes are stable or unstable for given parameters, instead of calculating the eigenvalues of the transfer matrix.

Figure 5 is a plot of $\hat{K}=K S / \varepsilon$ versus $\sigma_{v}$, showing the unstable regions of the slightly mismatched beam envelopes for $\eta=0.5$ and $g=0$ for three cases 


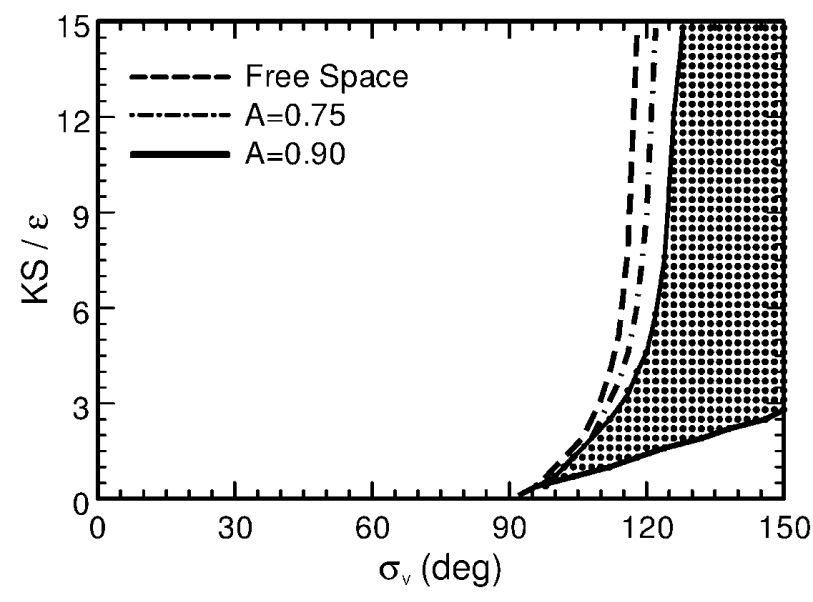

FIG. 5. Plot of the unstable regions in the dimensionless parameter space for the beam envelope evolution with $\eta=$ 0.5 and $g=0$ for three cases corresponding to (a) $R=\infty$ (free space), (b) $A=\tilde{a}_{0} / \hat{R}=0.75$, and (c) $A=\tilde{a}_{0} / \hat{R}=0.90$. Here, the shaded region is the unstable region for the beam envelope evolution with image-charge effects and $A=\tilde{a}_{0} / \hat{R}=0.90$.

corresponding to (a) $R=\infty$ (free space), (b) $A=$ $\tilde{a}_{0} / \hat{R}=0.75$ (with cylindrical conducting pipe), and (c) $A=\tilde{a}_{0} / \hat{R}=0.90$ (with cylindrical conducting pipe). As can be seen from Fig. 5, the unstable regions for all three cases start around $\sigma_{v}=90^{\circ}$. The solid lines indicate the boundary of the unstable region with image-charge effects from the cylindrical pipe for $A=\tilde{a}_{0} / \hat{R}=0.90$, whereas the dashed lines represent the boundary of unstable region without image-charge effects in free space. The unstable region with image-charge effects for $A=$ $\tilde{a}_{0} / \hat{R}=0.90$ is shaded in Fig. 5. Although the lower boundaries for the three cases almost coincide, there is an observable difference between their upper boundaries.

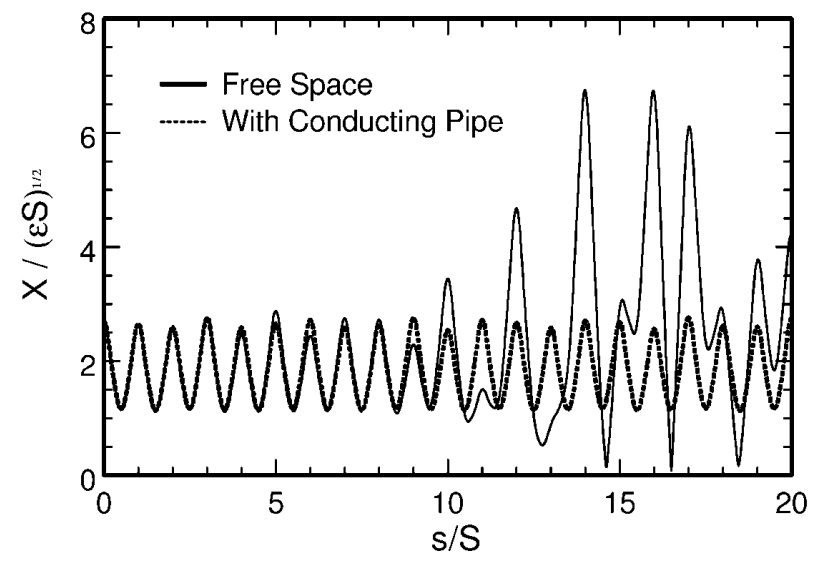

FIG. 6. Oscillations of the normalized beam envelope $\tilde{a}=$ $X / \sqrt{S \varepsilon}$ for both free-space and cylindrical conducting pipe cases with $\hat{K}=10.0, \sigma_{v}=120^{\circ}$, and $A=\tilde{a}_{0} / \hat{R}=0.9$, corresponding to a point in Fig. 5 where the envelopes in free space are unstable but the envelopes in cylindrical conducting pipe are stable.

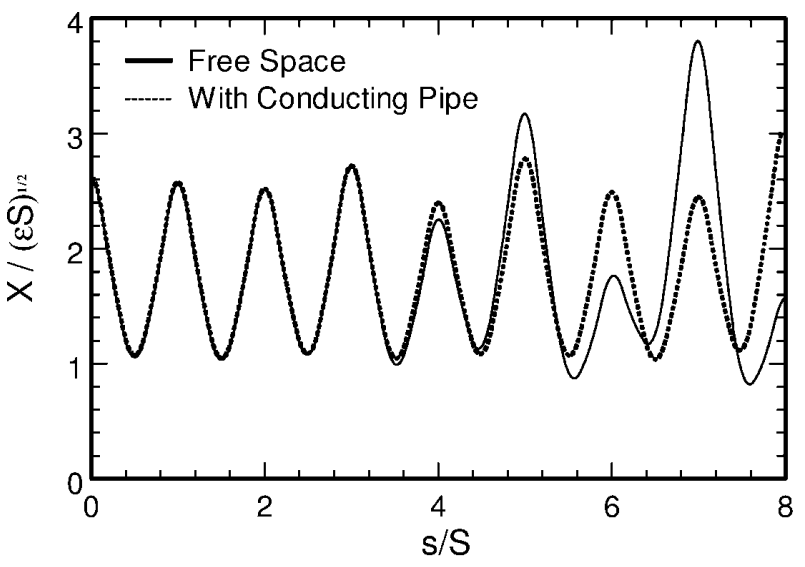

FIG. 7. Oscillations of the normalized beam envelope $\tilde{a}=$ $X / \sqrt{S \varepsilon}$ for both free-space and cylindrical conducting pipe cases at $\hat{K}=10.0, \sigma_{v}=130^{\circ}$, and $A=\tilde{a}_{0} / \hat{R}=0.9$, corresponding to a point in Fig. 5 where the envelopes are unstable in both free space and cylindrical pipe.

The unstable region for cylindrical pipe case (i.e., with image-charge effects) is obviously narrower than that in the free-space situation (i.e., without image-charge effects).

Figure 6 shows the oscillations of $\tilde{a}=X / \sqrt{S \varepsilon}$ for both free-space and cylindrical conducting pipe cases at $\hat{K}=$ $10.0, \sigma_{v}=120^{\circ}$, and $A=\tilde{a}_{0} / \hat{R}=0.9$, which corresponds to a point in Fig. 5 where the envelopes in free space are unstable but the envelopes in cylindrical conducting pipe are stable. Figure 7 shows the oscillations of $\tilde{a}=X / \sqrt{S \varepsilon}$ for both free-space and cylindrical conducting pipe cases at $\hat{K}=10.0, \sigma_{v}=130^{\circ}$, and $A=$ $\tilde{a}_{0} / \hat{R}=0.9$, which corresponds to a point in Fig. 5 where the envelopes are unstable in both free space and cylindrical conducting pipe. Figure 7 indicates that the unstable growth rate of the beam envelopes in free space is greater than that in cylindrical pipe. The results for free space agree with those obtained by Qian and Davidson in Ref. [11], in which they showed an unstable region for $90^{\circ}<\sigma_{v}<170^{\circ}$. It should be mentioned that the unstable regions are not sensitive to both $\eta$ and $g$ for the case of a cylindrical conducting pipe, which is similar to the fact that the instability in the free-space case is insensitive to $\eta$ as noted by Qian and Davidson [11].

\section{CONCLUSIONS}

In this paper, rms envelope equations were derived and analyzed for unbunched intense charged-particle beams in an alternating-gradient focusing field and a cylindrical metal pipe. All higher-order image-charge effects from the cylindrical pipe were expressed in terms of so-called multiple moment factors in the rms beam envelope equations, and the multiple moment factors were evaluated. Numerical results showed that for vacuum phase advance $\sigma_{v}<90^{\circ}$, the image-charge effects on the matched and slightly mismatched beam envelopes are negligibly 
small, at all orders, for all beams with arbitrary beam density profiles (including hollow density profiles) as well as for arbitrary small apertures (including beams with large aspect ratios). However, the main unstable region for the envelope evolution with image-charge effects, which occurs for $90^{\circ}<\sigma_{v}<270^{\circ}$, depending on the value of the normalized beam intensity $S K / \varepsilon$, was found to be narrower than its counterpart without image-charge effects.

\section{ACKNOWLEDGMENTS}

This research was supported by the U.S. Department of Energy, Office of Fusion Energy Science, Grant No. DEFG02-01ER54662, and Division of High-Energy Physics, Grant No. DE-FG02-95ER40919.

*Permanent address: Department of Applied Physics, National University of Defense Technology, Changsha 410073, Hunan, People's Republic of China.

[1] E. P. Lee and J. Hovingh, Fusion Technol. 15, 369 (1989).

[2] R.W. Müller, in Nuclear Fusion by Inertial Confinement: A Comprehensive Treatise, edited by $\mathrm{G}$. Velarde, Y. Ronen, and J. M. Martínez-Val (Chemical Rubber Co., Boca Raton, FL, 1993), Chap. 17, pp. 437-453.

[3] R. A. Jameson, in Advanced Accelerator Concepts, edited by J. S. Wurtele, AIP Conf. Proc. No. 279 (AIP, New York, 1993), p. 969.

[4] See, e.g., Space-Charge Dominated Beams and Applications of High-Brightness Beams, edited by S.Y. Lee, AIP Conf. Proc. No. 377 (AIP, New York, 1996).

[5] F. M. Bieniosek, J.W. Kwan, E. Henestroza, and C. Kim, Nucl. Instrum. Methods Phys. Res., Sect. A 464, 592 (2001)

[6] C. Chen and R. C. Davidson, Phys. Rev. Lett. 72, 2195 (1994).

[7] Q. Qian, R. C. Davidson, and C. Chen, Phys. Plasmas 2, 2674 (1995).
[8] Q. Qian, R. C. Davidson, and C. Chen, Phys. Plasmas 1, 1328 (1994)

[9] R.C. Davidson and Q. Qian, Phys. Plasmas 1, 3104 (1994).

[10] M. Reiser, Theory and Design of Charged-Particle Beams (Wiley, New York, 1994).

[11] Q. Qian and R.C. Davidson, Phys. Rev. E 53, 5349 (1996).

[12] I. Hofmann, L. Laslett, L. Smith, and I. Haber, Part. Accel. 13, 145 (1983)

[13] J. Stuckmeier, J. Klabunde, and M. Reiser, Part. Accel. 15, 47 (1984).

[14] R. Pakter and F. B. Rizzato, Phys. Rev. Lett. 87, 044801 (2001).

[15] R. Pakter and F. B. Rizzato, Phys. Rev. E 65, 056503 (2002).

[16] I. M. Kapchinskij and V.V. Vladimirskij, in Proceedings of the International Conference on High-Energy Accelerators and Instrumentation (CERN, Geneva, 1959), p. 274.

[17] P. Lapostolle, CERN Report No. AR/Int SG/65-15, 1965.

[18] F. J. Sacherer, IEEE Trans. Nucl. Sci. 18, 1105 (1971).

[19] P. Lapostolle, IEEE Trans. Nucl. Sci. 18, 1101 (1971).

[20] C. K. Allen and M. Reiser, Phys. Rev. E 54, 2884 (1996).

[21] C. K. Allen, Ph.D. thesis, University of Maryland, 1996.

[22] E. P. Lee, E. Close, and L. Smith, in Proceedings of the 1987 IEEE Particle Accelerator Conference, edited by E. R. Lindstorm and L. S. Taylor (IEEE, New York, 1987), p. 1126.

[23] R. L. Gluckstern, in Proceedings of the 1970 Proton Linear Accelerator Conference, edited by M. R. Tracy (National Accelerator Laboratory, Batavia, IL, 1971), p. 811.

[24] S. M. Lund and R. C. Davidson, Phys. Plasmas 5, 3028 (1998).

[25] R. Pakter and C. Chen, IEEE Trans. Plasma Sci. 28, 502 (2000).

[26] S. Yu, S. Eylon, E. Henestroza, and D. Grote, in SpaceCharge Dominated Beams and Applications of HighBrightness Beams (Ref. [4]). 